\title{
Genioplasty: evaluation and management
}

\author{
Ji Yun Choi, MD, $\mathrm{PhD}^{1}$, Chang Won Koh, MD² \\ ${ }^{1}$ Department of Otorhinolaryngology, Chosun University College of Medicine, Gwangju, Rep. of Korea, ${ }^{2}$ Department of Public Health Service, \\ Seoul National University Bundang Hospital, Seongnam, Rep. of Korea
}

\begin{abstract}
The secret to a beautiful face can be found in the overall harmony and balance of its anatomical units. It includes all anatomical units, such as teeth, bones, and soft tissues, influential on each other. The chin is one of the most prominent structures of the face, which plays a significant role in determining overall facial form. Prosthetic material graft is a simple procedure, while genioplasty is an effective procedure for correcting complex chin deformities. Sliding genioplasty offers a distinct advantage in that it gives surgeons the ability to resolve a host of chin abnormalities, ranging from underprojection, overprojection, vertical height disparities to transverse asymmetries.
\end{abstract}

Keywords: complications; genioplasty; horizontal osteotomy

\section{Introduction}

The secret to a beautiful face can be found in the overall harmony and balance of its anatomical units. It includes all anatomical units such as teeth, bones and, soft tissues, influential on each other [1]. The chin is one of the most prominent structures of the face, which plays a significant role in determining overall facial form. The size and location of the chin must be assessed by practitioners when evaluating if a patient is a good candidate for rhinoplasty.

The general public is not aware of the degree to which chin protrusion affects facial ratio. Most patients cannot recognize the degree of chin protrusion on their faces when they look at a front view image of themselves in the mirror. For this reason, non-severe chin deformation is usually ignored by patients. However, orthognathia has the potential to improve facial appearance. Hence, facial plastic surgeons must properly diagnose and treat chin deformations in order to maximize facial harmony and balance [2].

\section{Preoperative evaluation}

Precise analysis of facial skeletal structures and soft tissues is performed to determine the appropriate location of the chin. A physical examination and medical history of trauma, dental surgery and, orthodontic treatment on facial structures, such as lips, teeth, and other features is also performed. State of occlusion is recorded. Orthodontic treatment is conducted in patients with severe malocclusion, based on consultation with the orthodontist.

Three-dimensional analysis on the chin is necessary to determine the range of surgical chin-sliding. The effect of horizontal, vertical, and lateral location of the chin on other facial structures is explored. A pre-operative photo is taken of the front, lateral, and oblique views of face and a panorex x-ray is done to understand the location of the dental root and inferior alveolar nerve and mental foramen. Knowing these structural locations is critical to avoid damage during an osteotomy and descending fracture [3]. 


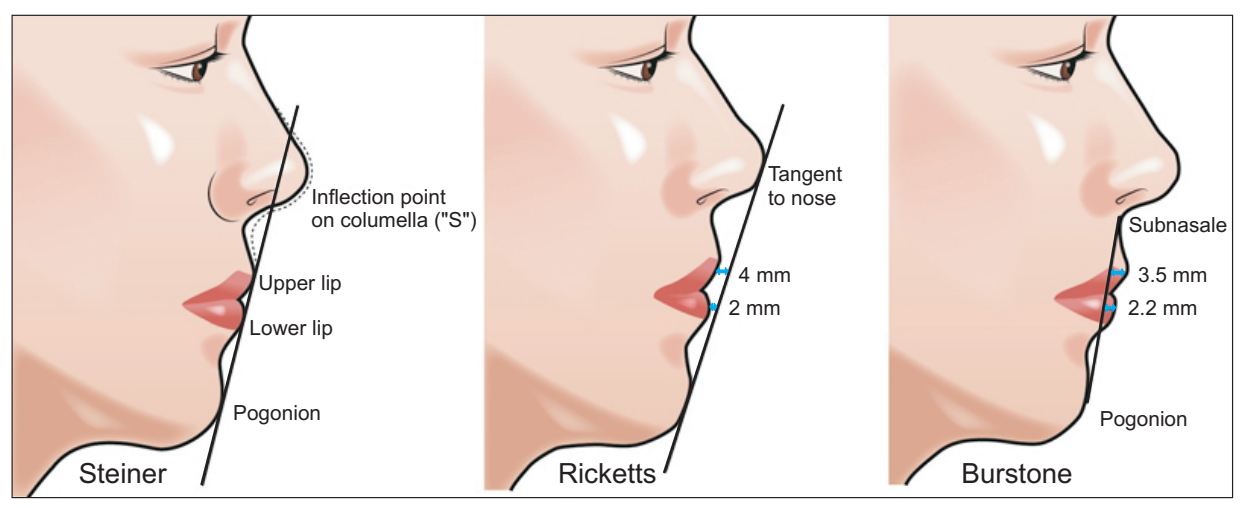

Fig. 1. Ideal lip position.

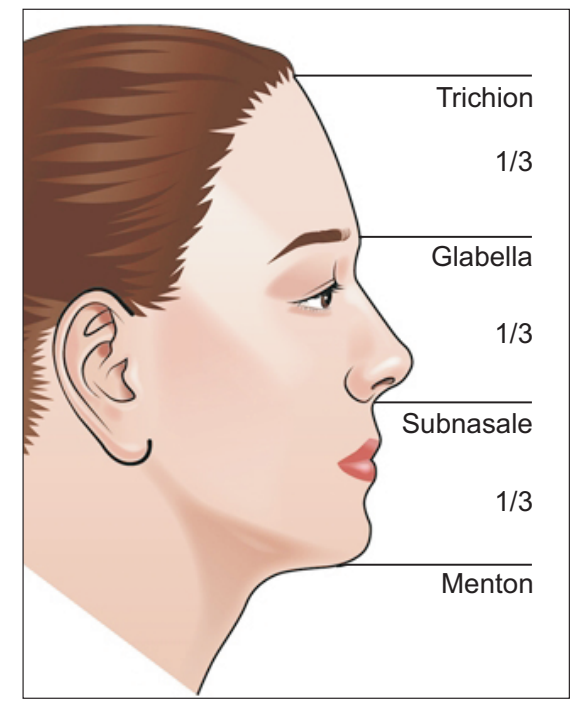

Fig. 2. Ideal vertical facial height.

X-ray is performed on the front and lateral sides of the head to precisely measure soft tissues and skeletal structure. These measurements are used to set the baseline and orientation angle. Diagnosis and treatment of maxillofacial deformity is performed via various analyses using the measurements mentioned above, baseline and orientation angle [4]. In particular, the correlation between the pogonion (the most protruding part of the chin) and other facial structures such as the upper lip, lower lip and tip of the nose is analyzed [5]. Steiner [5], Ricketts [6], Burstone [7], González-Ulloa and Stevens [8] suggest a standard for locating the ideal mentum. For example, Ricketts [6] argues that ideally, the upper lip and lower lip are located 4 $\mathrm{mm}$-posterior and $2 \mathrm{~mm}$ posterior from the line drawn between the pogonion and the tip of the nose, respectively. The analysis by Ricketts [6] does not match the relationship between the lower facial structures, but it is significant in analyzing the ideal location of the tip of the nose. There is no single, perfect method to assess the ideal location of the chin although a number of

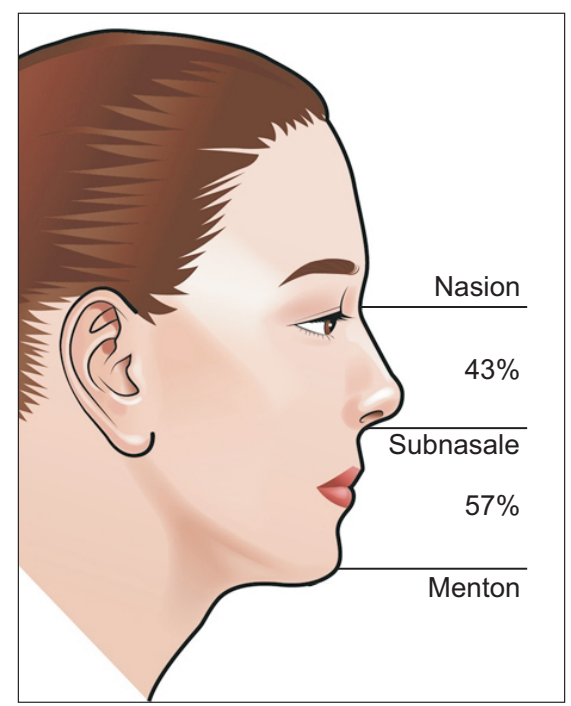

Fig. 3. Ideal chin ration by Dr. Powell.

methods have been explored (Fig. 1).

Compared to the length of other facial structures, the vertical length of the chin is critical for facial esthetics. Sometimes, practitioners overlook the vertical length of the chin while focusing on its horizontal protrusion. One of the most common chin deformities is vertical overgrowth horizontal development [9]. Orthognathic procedures using implants that overlook such vertical overgrowth may cause more vertical overgrowth.

Several methods to assess the vertical length of the chin have been introduced. One of the most commonly used methods involves tri-sectioning the face (Fig. 2). In an effort to modify the tri-sectioning method, Powell and Humphreys [10] showed that the middle of the face ( $1 / 3$ ) (from nasion to subnasale) took up $43 \%$ of the lower face $(2 / 3)$ with no consideration for the upper face (1/3), i.e., different headlines depending on the person (Fig. 3).

Another important factor in assessing the vertical length of the lower face is parted lips upon rest. Ideally, the front teeth of 
the upper jaw should be 0 to $3 \mathrm{~mm}$ protruded. More than $3 \mathrm{~mm}$ protrusion may involve vertical overgrowing of mid face. The result of the vertical length analysis reveals that the distance from the subnasale to the cutaneous mucosal boundary line of the lower lip is consistent with the distance from the cutaneous mucosal boundary line of lower lip to then menton. In addition, the distance from the subnasale to the stomion corresponds to half of the distance from the stomion to the menton [11]. Horizontal and vertical length analysis as well as lateral length analysis must be conducted. Bilateral asymmetry of the chin, if any, should be discussed with the patient before surgery. Chin symmetry may go unrecognized if there is facial disfigurement, such as hemifacial microsomia. However, it can be assessed by comparing facial measurements such as the centerline of the chin and teeth and the centerline of the upper and lower jaw, using an X-ray for head measurement. It is crucial to diagnose and record chin asymmetry before surgery. Orthognathic procedures using prosthesis can make previous asymmetry more pronounced in the case of an undeveloped chin with vertical and bilateral asymmetry.

\section{Select surgical procedure}

Selection of a surgical procedure to correct chin deformity depends on the intensity and shape of the deformity. Use of prosthesis is a simple and reliable approach in the case of a moderately undeveloped chin. However, prosthetic use has some limitations. It is difficult to correct vertical deformity or bilateral asymmetry of the chin using prosthesis. In fact, most prosthesis exacerbate the asymmetry and vertical deformity of the chin [12]. Genioplasty is another surgical procedure used to move the mental bone three-dimensionally in any direction. It was initially introduced by Hofer [13] in 1942. Horizontal os- teotomy and descending fracture are conducted at the inferior mental nerves after exposing the mental bone through an intraoral incision [13]. With genioplasty, practitioners can move back and forth in the anterior and posterior direction, which helps them achieve vertical extension and a reduction in chin length. Genioplasty can also help correct bilateral asymmetry [14].

The selection of genioplasty or prosthetic graft material is determined based on the shape of the chin deformity or the practitioner's preference. Prosthetic grafting is a relatively simple procedure, while genioplasty is effective for correcting chin deformities with complex shapes.

\section{Surgical procedure}

Horizontal osteotomy can be conducted on the chin under

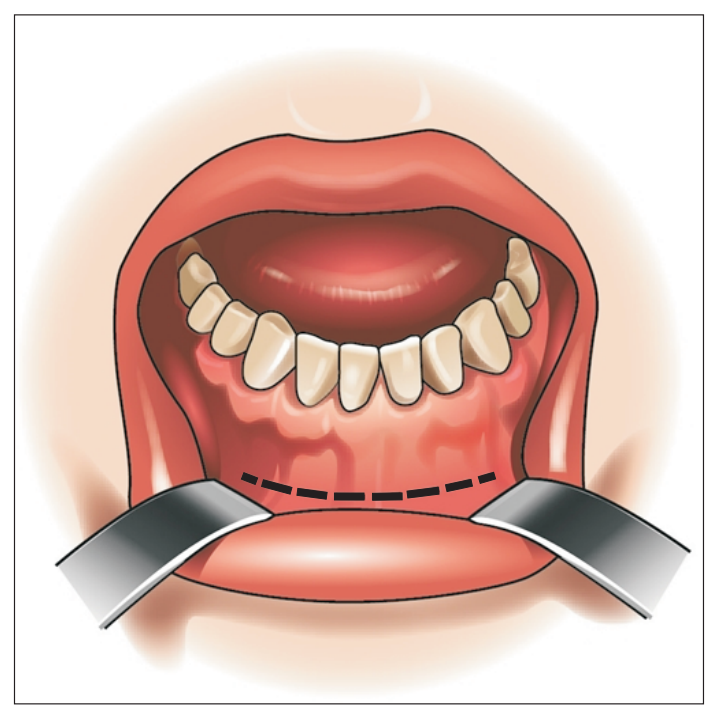

Fig. 4. Illustration of intraoral gingival incision for genioplasty.
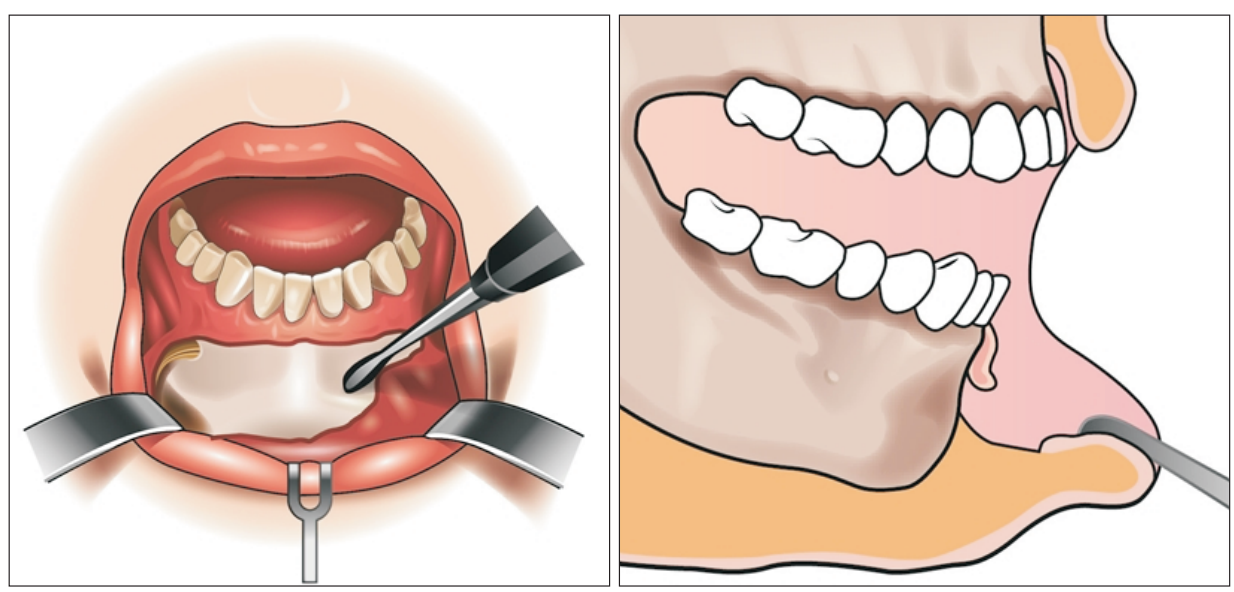

Fig. 5. Illustration of subperiosteal dissection. Identification and preservation of mental nerve are shown bilaterally. 
general anesthesia or partial anesthesia using a nerve block. Orotracheal intubation is conducted with concurrent rhinoplasty, while nasotracheal intubation is conducted without rhinoplasty.

An intraoral gingivobuccal incision is made between bilateral canine teeth (Fig. 4). Dissection is conducted until the periosteum of the lower jaw is reached. When incision is conducted at the lips followed with gingivobuccal groove to remain sufficient mucoperiosteal flap for wound closure. Subperiosteal dissection is conducted while checking and preserving bilateral mental nerves. Dissection is conducted at the inferior and external mental foramens to secure sufficient space for an osteotomy beneath the mental nerves (Fig. 5).

The centerline is marked by using the side-cutting bar upon completion of subperiosteal dissection. It is necessary to precisely locate bone fragments in the desirable place after osteotomy. Vertical osteotomy is designed and marked using a caliper (Fig. 6). This marking is critical to ensure that osteotomy is not done asymmetrically. Osteotomy should be performed at least $5 \mathrm{~mm}$ beneath the mental foramen. This is to prevent any nerve damage, since the inferior alveolar canal is located 2 to $3 \mathrm{~mm}$ beneath the mental foramen.

Vertical osteotomy on the lower jaw is done using the recip-

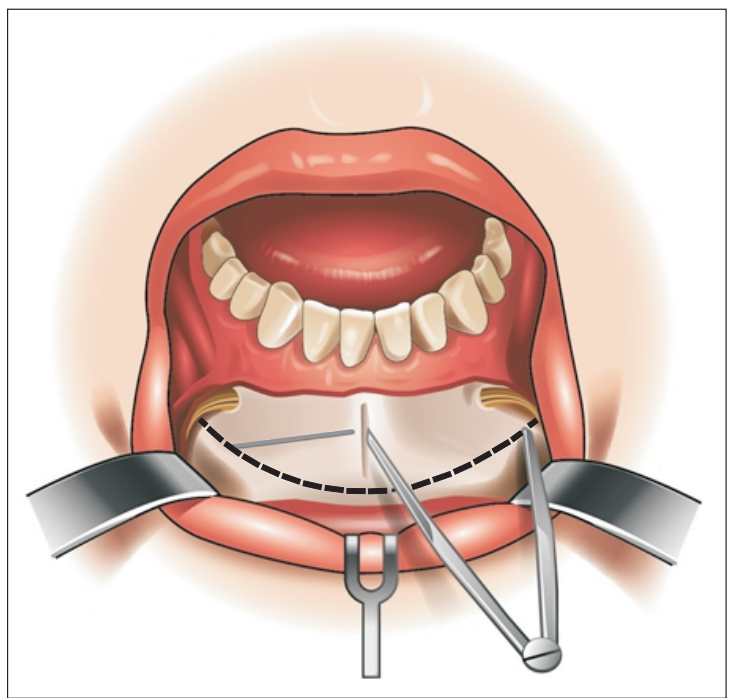

Fig. 6. Design of horizontal osteotomy.
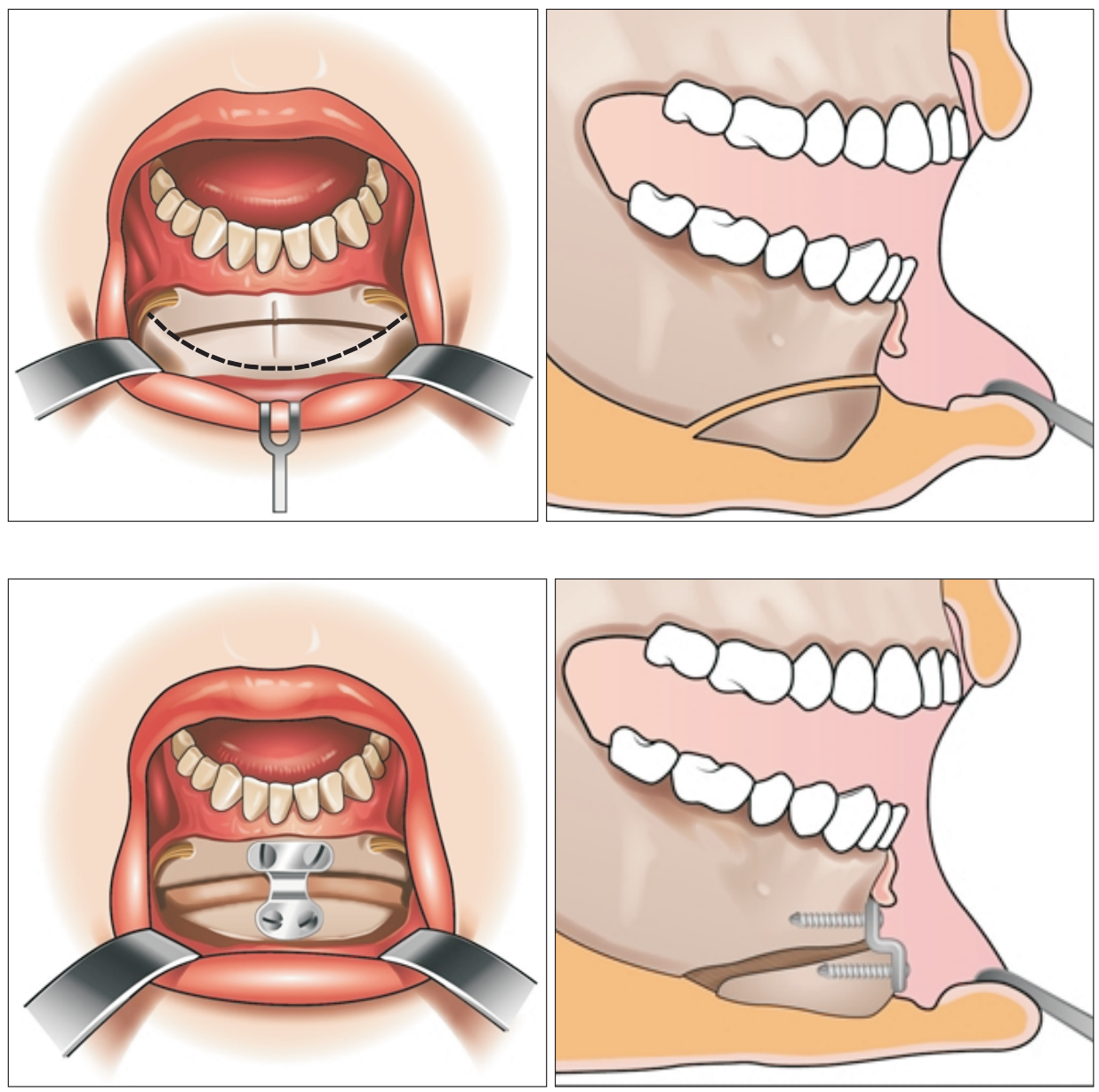

Fig. 7. Illustration of horizontal osteotomy.

Fig. 8. Illustration shows horizontal advancement and plate fixation of chin. 
rocating saw. At this time, caution must be taken not to damage the mental nerves (Fig. 7). During pre-operative assessment, the degree of shift in the chin should be determined. Spot of osteotomy and shift of the chin is determined according to the surgical plan. Osteotomy conducted diagonally causes the vertical length of the chin to be reduced in proportion to the forward distance that the chin moves. Osteotomy should be conducted horizontally to move the chin back and forth. If the backwards and forward movement of the chin as well as the reduction of its vertical length are the desirable outcomes, then osteotomy should be done diagonally.

After marking the osteotomy, the mental nerves and lips are cautiously pulled away to prevent nerve damage. Osteotomy is then done from the inside out using the reciprocating saw. Osteotomies conducted bilaterally and from the inside out respectively, meet at the center. Distal fracture is done in a downward direction using finger pressure. A small amount of soft tissue was sharply dissected from the tongue side of lower jaw to make perfect distal movement.

The distal lower jaw is relocated according to the pre-operative plan. The degree of horizontal forward movement is adjusted using the solid, fixed plate manufactured pre-operatively. A temporary screw is then applied to the distal part of the plate to position it in place. An additional fixed plate is applied if the solid, fixed plate at the center fails to maintain appropriate stability (Fig. 8). Any correction will be made before fixation if necessary in order to change the vertical height of the lower face $(1 / 3)$. An increase in vertical length is accomplished by placing the autograft or artificial bone between bone fragments. A decrease in vertical length is accomplished by conducting a secondary horizontal osteotomy to remove the bone fragment in the middle.

Upon completion of the osteotomy, shift and fixation, soft tissues of the lips and chin are placed at the original location and the new contour of the chin is assessed. Revisions are then made if any asymmetry or imbalance, is observed. If the result of the assessment is satisfactory, the mentalis muscle is sutured in two layers using the 4-0 vicryl. This is crucial to prevent soft tissues from getting loose after surgery. The mucous membrane is sutured in running locking form using the 4-0 vicryl. Chinstrap pressure dressing is done for five days. Antibiotic agent is administered for 48 hours and a soft diet is observed by the patient for two weeks.

\section{Complications}

Some complications of genioplasty include bleeding, infection and opening of intraoral scars. Infection and bleeding are rare when the appropriate bleeding control and aseptic clinical steps are taken. Mental nerve damage and nonunion and wrong distal fixation are also possible complications. Preoperative three-dimensional planning should be done thoroughly to reduce any further complications. Osteotomy must be performed precisely according to the pre-operative plan and fixation should be done at the correct location. Suturing should be conducted with caution and by layer to prevent soft tissues of the chin from getting loose after surgery [15-18].

\section{Conclusion}

It is a well-established fact that the secret to a beautiful face can be found in the overall harmony and balance of its anatomical units. In evaluating the profile of patients who seek facial cosmetic enhancement, the published norms of facial ratios and radiographic cephalometric measurements should serve as valuable guides in determining midface and lower face proportions. Among the various procedures, chin implant remains the most commonly used, but sliding genioplasty offers a viable alternative to alloplastic mentoplasty to correct chin retrusion. Sliding genioplasty offers a distinct advantage in that it gives surgeons the ability to resolve a host of chin abnormalities, ranging from underprojection, overprojection, vertical height disparities to transverse asymmetries.

\section{Conflicts of interest}

The authors have nothing to disclose.

\section{References}

1. Lehman JA Jr. Soft-tissue manifestations of aesthetic defects of the jaws: diagnosis and treatment. Clin Plast Surg 1987;14:767-83.

2. Downs WB. Variations in facial relationships; their significance in treatment and prognosis. Am J Orthod 1948;34:81240.

3. Spear SL, Kassan M. Genioplasty. Clin Plast Surg 1989;16:695706.

4. Rakosi T. An atlas and manual of cephalometric radiography. Philadelphia, PA: Lea \& Febiger; 1982. 
5. Steiner CC. Cephalometrics in clinical practice. Angle Orthod 1959;29:8-29.

6. Ricketts RM. Esthetics, environment, and the law of lip relation. Am J Orthod 1968;54:272-89.

7. Burstone CJ. Lip posture and its significance in treatment planning. Am J Orthod 1967;53:262-84.

8. González-Ulloa M, Stevens E. Role of chin correction in profileplasty. Plast Reconstr Surg 1968;41:477-86.

9. Precious DS, Delaire J. Correction of anterior mandibular vertical excess: the functional genioplasty. Oral Surg Oral Med Oral Pathol 1985;59:229-35.

10. Powell N, Humphreys B. Proportions of the aesthetic face. New York: Thieme-Stratton; 1984.

11. Bell WH, Gallagher DM. The versatility of genioplasty using a broad pedicle. J Oral Maxillofac Surg 1983;41:763-9.

12. McCarthy JG, Ruff GL. The chin. Clin Plast Surg 1988;15:125-
37.

13. Hofer O. [Operation der prognathie und mikrogenie]. Dtsch Zahnarztl Mund Kieferheilkd 1942;9:121-32. German.

14. Converse JM, Wood-Smith D. Horizontal osteotomy of the mandible. Plast Reconstr Surg 1964;34:464-71.

15. Sykes J, Donald PJ. Orthognathic surgery. In: Papel ID, Nachlas NE, editors. Facial plastic and reconstructive surgery. St. Louis, MO: Mosby; 1992.

16. Sykes J, Frodel JL. Mentoplasty. In: Cummings CW, editor. Otolaryngology - head and neck surgery. 2nd ed. St. Louis, MO: Mosby Year Book; 1992.

17. Sykes JM, Frodel JL Jr. Genioplasty. Oper Tech Otolaryngol 1995;6:319-23.

18. Wolfe SA. The chin. In: Wolfe SA, Berkowitz S, editors. Plastic surgery of facial skeleton. Boston, MA: Little, Brown and Company; 1989. 